\title{
Conversational Case-Based Reasoning
}

\author{
David W. Aha \\ Head, Intelligent Decision Aids Group, \\ Navy Center for Applied Research in Artificial Intelligence, \\ Naval Research Laboratory (Code 5515), \\ Washington, DC 20375 \\ david.aha@nrl.navy.mil
}

\section{Overview}

Case-based reasoning (CBR) is a problem solving methodology that focuses on reusing lessons obtained from previous (possibly generalized) experiences towards solving new problems (Kolodner, 1993; Aamodt \& Plaza, 1994; Watson, 1999; Bergmann, 2002). Originally conceived by cognitive scientists, since 1993 the CBR community has focused primarily on issues of interest to artificial intelligence researchers and practitioners. Some research topics of particular interest include case representation and indexing, solution retrieval and adaptation, learning (e.g., case acquisition), and integrating case-based approaches with others. Some motivating applications have included those related to customer support, recommender systems, knowledge management, diagnosis, the health sciences, and legal reasoning.

Among these areas, most deployed CBR applications have been for the customer support niche of help-desk systems. These applications have the characteristic that problem descriptions are incrementally elicited from a user rather than available in complete form a priori. This category of $\mathrm{CBR}$ approaches is referred to as conversational (Aha et al., 2001) because they use a dialogue with the end user to identify the problem to be solved. Many case-based diagnosis and recommendation systems have used a conversational CBR (CCBR) methodology, and met with considerable success.

In this presentation, I will introduce the CCBR methodology, describe its evolution, examine its various manifestations and their motivations, highlight recent work, and describe unsolved issues that could be the focus of enlightened dissertations.

\section{References}

Aamodt, A., \& Plaza, E. (1994). Case-based reasoning: Foundational issues, methodological variations, and system approaches. AI Communications, 7, 39-59.

Aha, D.W., Breslow, L.A., \& Munoz-Avila, H. (2001). Conversational case-based reasoning. Applied Intelligence, 14(1), 9-32.

Bergmann, R. (2002). Experience management: Foundations, development methodology, and Internet-based applications. Berlin: Springer.

Kolodner, J. (1993). Case-based reasoning. San Mateo, CA: Morgan Kaufmann.

Watson, I., (1999). CBR is a methodology not a technology. Knowledge Based Systems Journal, 12(5-6), 303-308. 